\title{
Superconducting Solenoid System with Adjustable Shielding Factor for Precision Measurements of the Properties of the Antiproton
}

\author{
Jack A. Devlin $\odot,{ }^{1,2, *}$ Elise Wursten,,${ }^{1,2, \dagger}$ James A. Harrington, ${ }^{1,3}$ Takashi Higuchi, ${ }^{1,4,+}$ \\ Pascal E. Blessing, ${ }^{1,5}$ Matthias J. Borchert, ${ }^{1,6}$ Stefan Erlewein, ${ }^{1,2,3}$ Jannek J. Hansen, ${ }^{1}$ \\ Jonathan Morgner, ${ }^{1,6}$ Matthew A. Bohman, ${ }^{1,3}$ Andreas H. Mooser, ${ }^{1,3}$ Christian Smorra, ${ }^{1}$ \\ Markus Wiesinger, ${ }^{1,3}$ Klaus Blaum, ${ }^{3}$ Yasuyuki Matsuda,${ }^{4}$ Christian Ospelkaus, ${ }^{6,7}$ Wolfgang Quint, ${ }^{5}$ \\ Jochen Walz, ${ }^{8}$ Yasunori Yamazaki, ${ }^{1}$ and Stefan Ulmer ${ }^{1}$ \\ ${ }^{1}$ RIKEN, Ulmer Fundamental Symmetries Laboratory, 2-1 Hirosawa, Wako, Saitama 351-0198, Japan \\ ${ }^{2}$ CERN, Esplanade des Particules 1, CH-1211 Geneva 23, Switzerland \\ ${ }^{3}$ Max-Planck-Institut für Kernphysik, Saupfercheckweg 1, D-69117 Heidelberg, Germany \\ ${ }^{4}$ Graduate School of Arts and Sciences, University of Tokyo, 3-8-1 Komaba, Tokyo 153-8902, Japan \\ ${ }^{5}$ GSI-Helmholtzzentrum für Schwerionenforschung GmbH, Planckstraße 1, D-64291 Darmstadt, Germany \\ ${ }^{6}$ Institut für Quantenoptik, Leibniz Universität Hannover, Welfengarten 1, D-30167 Hannover, Germany \\ ${ }^{7}$ Physikalisch-Technische Bundesanstalt, Bundesallee 100, D-38116 Braunschweig, Germany \\ ${ }^{8}$ Institut für Physik, Johannes Gutenberg-Universität, Staudinger Weg 7, D-55128 Mainz, Germany
}

(Received 9 July 2019; revised manuscript received 27 August 2019; published 4 October 2019)

\begin{abstract}
A superconducting self-shielding three-solenoid system with an adjustable shielding factor is developed, implemented, and characterized using a single antiproton in a Penning trap. With the tuned system, we suppress external magnetic field disturbances by up to a factor of $225 \pm 15$, allowing antiproton-toproton charge-to-mass ratio comparisons with fourfold reduced frequency fluctuations and antiproton magnetic moment determinations with tenfold reduced uncertainty.
\end{abstract}

DOI: 10.1103/PhysRevApplied.12.044012

\section{INTRODUCTION}

Single particles in Penning traps are sensitive probes to test fundamental symmetries and to measure fundamental constants. Stringent tests of charge-parity-time-reversal symmetry [1-4] and quantum electrodynamics [5] have been performed with such systems, as well as the most precise determinations of masses in atomic units [6-8] and the fine-structure constant $\alpha$ [9].

A great strength of the Penning trap is its conceptual simplicity. A stable static magnetic field and an electrostatic potential define the trap volume [10], in which single particles can be stored and observed for years [11]. The fields, together with particle properties such

\footnotetext{
*jack.alexander.devlin@cern.ch

†elise.wursten@cern.ch

¥Present affiliation: Research Center for Nuclear Physics, Osaka University, 10-1 Mihogaoka, Ibaraki, Osaka 567-0047, Japan.

Published by the American Physical Society under the terms of the Creative Commons Attribution 4.0 International license. Further distribution of this work must maintain attribution to the author(s) and the published article's title, journal citation, and DOI.
}

as the charge $q$, mass $m$, or magnetic moment $\mu$, lead to characteristic oscillation frequencies. Direct measurements of these frequencies by weakly coupled detection circuits with single-particle sensitivity [12] give access to the fundamental properties of the particle. Measurements of cyclotron frequencies $v_{c}$ enable the determination of charge-to-mass ratios $q / m$, while $g$ factors can be extracted from the ratio of the Larmor frequency $v_{L}$ to the cyclotron frequency $g / 2=v_{L} / v_{c}$. The most precise Penning-trap charge-to-mass ratio comparisons reach 7 parts per trillion (p.p.t.) $[13,14]$, and the best $g$-factor measurements reach 0.3 p.p.t. [9].

A limitation of Penning traps is that any external fluctuations that interfere with the trapping fields add noise to the frequency measurements. A particular challenge is that Penning-trap experiments on exotic particles are often located in environments where the magnetic field noise is especially high, as the particles have to be produced at powerful accelerator facilities such as CERN's Antiproton Decelerator [15], radioactive ionbeam sources [16-21], or highly charged ion facilities [22]. Without effective strategies to combat this noise, Penning-trap-based frequency measurements struggle to reach uncertainties on the parts-per-billion (p.p.b.) level or below. 
In this paper, we describe an innovative general approach to efficiently shield the Penning trap of the BASE experiment [23] at CERN from magnetic field fluctuations imposed by the Antiproton Decelerator (AD). We create a multicoil system of persistent superconducting coils that allows optimization of the shielding factor by selective coil activation. With the coil system active, we suppress certain external magnetic field disturbances by a factor of approximately $225 \pm 15$. By combining this multicoil self-shielding system with a data-acquisition sequence linked to the deceleration cycle of the $\mathrm{AD}$, we suppress the parts-per-million-level magnetic field fluctuations and measure antiproton cyclotron-frequency fluctuations of $1.5 \pm 1$ p.p.b., where the suppressed external magnetic field fluctuations are presently not resolved.

\section{MAGNETIC ENVIRONMENT}

To illustrate the operational conditions in CERN's AD facility, Fig. 1(a) shows the typical magnetic field noise over $24 \mathrm{~h}$ along $\hat{\mathbf{z}}$, the direction of the horizontal superconducting magnet of $B_{m}=1.945 \mathrm{~T}$ used in the BASE setup. Numerous features are present, including the switching of the $\mathrm{AD}$ extraction magnets to steer the antiproton beam into a neighboring experimental zone and the magnetic disturbance produced when a bridge crane moves over

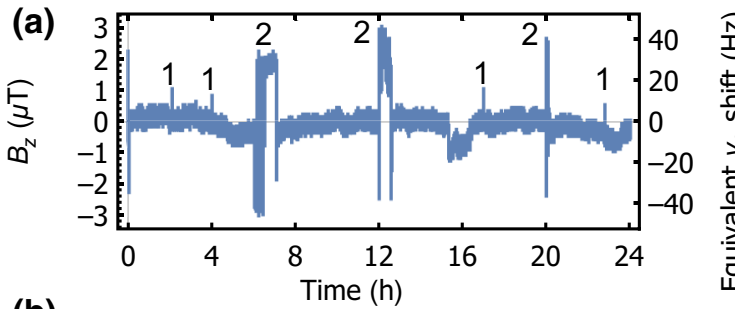

(b)

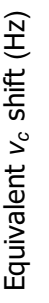

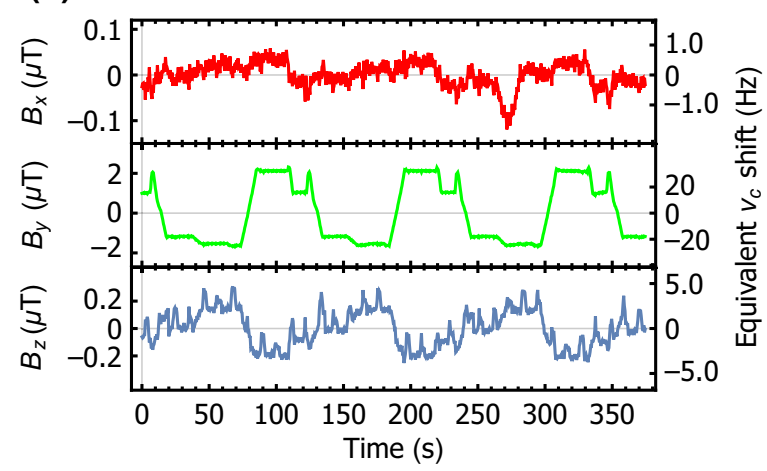

FIG. 1. Magnetic field changes in the BASE experiment zone at CERN. (a) The magnetic field along the magnet axis measured for $24 \mathrm{~h}$ by a Hall probe on the superconducting magnet. The labeled events are as follows: $1, \mathrm{AD}$ extraction magnets switching; 2, bridge crane moving over the BASE zone. (b) The AD magnetic field along the three axes as measured by a flux-gate sensor. The $x$ axis is horizontal and perpendicular to the magnet, the $y$ axis is vertical, and the $z$ axis is oriented along the horizontal magnet axis. the BASE trap. Other magnetizable objects moving in the fringe field of the magnet can also cause field changes. Moreover, the ramping of the magnetic field every $\mathrm{AD}$ cycle creates periodic magnetic field changes, as shown in Fig. 1(b). The largest field shift, $>3 \mu \mathrm{T}$, is in the vertical direction $\hat{\mathbf{y}}$, whereas shifts on the order of several 100 nT (50 p.p.b.) per $120 \mathrm{~s}$ deceleration cycle are observed along the horizontal trap axis. The ELENA decelerator [24], with its closest magnet located approximately $5 \mathrm{~m}$ from the BASE experiment, also adds 30-90 nT peak-topeak fluctuations with a period of $10-90 \mathrm{~s}$. Unshielded, these fluctuating fields add unacceptable noise, corresponding to peak-to-peak fluctuations in the instantaneous cyclotron frequency $(29.6 \mathrm{MHz})$ of approximately $8 \mathrm{~Hz}$ (270 p.p.b.) for a horizontal superconducting solenoid field as in BASE and approximately $60 \mathrm{~Hz}(2000$ p.p.b.) if the solenoid is vertically oriented. The self-shielding multicoil system presented in this paper is invented to efficiently damp these external fluctuations with high flexibility and to enable measurements of the fundamental properties of the antiproton at the sub-parts-per-billion level.

\section{THE SELF-SHIELDING COIL}

The self-shielding coil (SSC) was invented to remove external magnetic field noise in a cryogenic experiment. It was pioneered by Gabrielse and Tan [25] and is further developed in this work. In its simplest form, this consists of a closed persistent superconducting solenoid of a carefully chosen length-to-diameter $(l / d)$ ratio, which is introduced into the Penning-trap system. For the optimum choice of $l / d$, conservation of magnetic flux ensures that the persistent current induced in the self-shielding coil generates a magnetic field $B_{\mathrm{SSC}} \hat{\mathbf{c}}$ at the particle position that exactly cancels the external magnetic field changes along $\hat{\mathbf{c}}$.

A schematic diagram of a section of the coil, the Penning trap, and the magnetic fields at the particle position $\mathbf{x}_{0}$ inside the SSC is shown in Fig. 2. From these fields, we can define the intrinsic shielding factor $S$ as follows:

$$
S^{-1}=\frac{\Delta \mathbf{B}_{e} \cdot \hat{\mathbf{c}}+B_{\mathrm{SSC}}}{\Delta \mathbf{B}_{e} \cdot \hat{\mathbf{c}}}
$$

Here, $\Delta \mathbf{B}_{e}$ is the external-field change at $\mathbf{x}_{0}$. For an ideal densely wound single-layer self-shielding solenoid, choosing $l / d \approx 0.8760$ perfectly shields homogeneous external magnetic field disturbances parallel to the coil axis for a particle at the coil center.

In practice, achieving a large shielding factor $S>100$ involves overcoming a range of imperfections and perturbing effects. A significant difficulty in designing coils with very high shielding factors is the effect of mutual inductance between the shielding coil, the main superconducting 


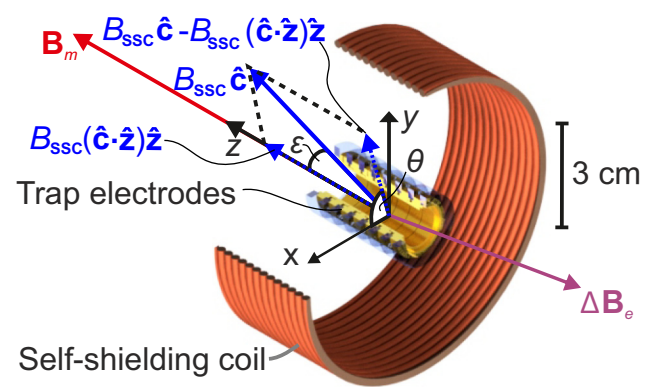

FIG. 2. A diagram showing the Penning-trap electrodes and a section of the self-shielding coil. To include the effect of a misalignment of the coil, discussed in the main text, we show the vector direction of the magnetic fields at the particle position $\mathbf{x}_{0}$ produced by the superconducting magnet $B_{m} \hat{\mathbf{z}}$, the coil $B_{\mathrm{SSC}} \hat{\mathbf{c}}$, and an arbitrarily orientated external magnetic field change $\Delta \mathbf{B}_{e}$, together with the angles $\varepsilon$ and $\theta$.

coil, and the numerous shim coils of the Penning-trap magnet. Considering, for simplicity, the case of a single exterior coil: if the SSC is perfectly centered inside the coil, the effect on the optimum $l / d$ is only significant when the coupling constant $\kappa=M / \sqrt{L_{\mathrm{SSC}} L_{\mathrm{EC}}}$ approaches $\kappa=1$. Here, $L_{\mathrm{SSC}}$ and $L_{\mathrm{EC}}$ are the self-inductance of the SSC and the exterior coil, respectively, and $M$ is the mutual inductance between the coils (all inductance calculations in this work use the formulas given in Ref. [26]). For example, an SSC with $l / d=0.8760$ placed inside a superconducting coil of the same length and $25 \%$ larger diameter $(\kappa=0.72)$ leads to a coupled system with a combined shielding factor $S=-101$. To achieve perfect shielding inside such an exterior coil, the interior coil should have $l / d=0.8413$, which is $4 \%$ smaller than the value for the free selfshielding solenoid. If the SSC and the exterior coil are concentric but axially displaced, the optimum $l / d$ value can shift more significantly, even if $\kappa \ll 1$. Since the dimensions and locations of the coils inside a commercial superconducting magnet are not generally known, these effects can only be approximately estimated during the design of a self-shielding solenoid.

A second important consideration is the effect of imperfections in the manufacturing and installation of the SSC. The shielding factor is very sensitive to the coil dimensions and position. For small deviations $\delta(l / d)$ about the optimum $l / d$ value, the inverse shielding factor for a densely wound single-layer solenoid scales as $\delta\left(S^{-1}\right) \approx$ $-0.25 \delta(l / d)$, so that a $2-\mathrm{mm}$ error in the length on a coil of nominal length $68 \mathrm{~mm}$ and nominal diameter $78 \mathrm{~mm}$ limits the shielding factor to $|S|<160$. For various technical reasons, it is difficult to achieve a significantly lower uncertainty on the overall coil length. In addition, the position of the SSC with respect to the particle position $\mathbf{x}_{0}$ modifies the shielding factor or, equivalently, the required $l / d$ ratio for optimal shielding. For example, a shift of
$2 \mathrm{~mm}$ with respect to the foreseen particle position in the previously mentioned coil typically limits the shielding factor to $|S|<120$. Spatial constraints mean that it is not always possible to align the SSC center with the particle position, so having a larger volume with a high shielding factor is beneficial.

The performance of the SSC system is also strongly affected by any angle $\varepsilon$ between the SSC axis $\hat{\mathbf{c}}$ and the main solenoid axis $\hat{\mathbf{z}}$, as depicted in Fig. 2. This can be caused by a tilt in the coil windings or because the entire SSC coil is tilted relative to the magnet bore. To understand why this is a problem, we start by writing the total field at the position of the particle as follows:

$$
\mathbf{B}_{\mathrm{tot}}=B_{m} \hat{\mathbf{z}}+\Delta \mathbf{B},
$$

where

$$
\begin{aligned}
\Delta \mathbf{B} & =\Delta \mathbf{B}_{e}+B_{\mathrm{SSC}} \hat{\mathbf{c}} \\
& =\Delta \mathbf{B}_{e}+S^{-1}\left(\Delta \mathbf{B}_{e} \cdot \hat{\mathbf{c}}\right) \hat{\mathbf{c}}-\left(\Delta \mathbf{B}_{e} \cdot \hat{\mathbf{c}}\right) \hat{\mathbf{c}} .
\end{aligned}
$$

Here, we use (1) to rewrite $B_{\text {SSC. Since }} B_{m} \gg\left|\Delta \mathbf{B}_{e}\right|$, only the change in the $z$ component of the magnetic field significantly changes the magnitude of the total field and hence the cyclotron frequency of the trapped particle. Therefore, we define a effective shielding factor $S_{\text {eff }}$

$$
S_{\mathrm{eff}}^{-1}=\frac{\Delta \mathbf{B} \cdot \hat{\mathbf{z}}}{\Delta \mathbf{B}_{e} \cdot \hat{\mathbf{z}}}
$$

Note that this effective shielding factor depends on the direction of $\Delta \mathbf{B}_{e}$. Using $\cos \varepsilon=\hat{\mathbf{c}} \cdot \hat{\mathbf{z}}$, we find, to first order in $\varepsilon$, that

$$
S_{\text {eff }}^{-1}=S^{-1}-\varepsilon \frac{\Delta B_{e, x} \cos \theta+\Delta B_{e, y} \sin \theta}{\Delta B_{e, z}}\left(1-S^{-1}\right) .
$$

Here, $\Delta B_{e, x}, \Delta B_{e, y}$, and $\Delta B_{e, z}$ are the three components of the external-field change and $\theta$ is the angle between the projection of $\hat{\mathbf{c}}$ onto the $x-y$ plane and the $x$ axis, as shown in Fig. 2. The effect of a tilted coil and an angled field can lead to a pronounced difference between the effective and intrinsic shielding factors. As an example, if $\varepsilon=1^{\circ}$, then a coil with $S^{-1}=0$ will have an effective shielding factor of $S_{\text {eff }}<100$ with respect to $67 \%$ of all possible external magnetic field directions. In addition, if the misalignment angle $\varepsilon$ of the SSC fluctuates by $\delta \varepsilon$ - for example, due to vibrations - this induces fractional magnetic field noise $\delta B / B_{m}$ parallel to $\hat{\mathbf{z}}$, which is described to first order in $\varepsilon$ by the following:

$$
\delta B / B_{m}=\left(1-S^{-1}\right) \varepsilon \delta \varepsilon .
$$

Hence it is crucial to keep $\varepsilon$ as close to zero as possible. 
These considerations make it difficult to ensure that an optimally designed SSC system will effectively yield $S_{\text {eff }}>100$ when constructed and installed in an existing superconducting magnet. The approach described in this paper overcomes this difficulty with through the development of a self-shielding coil system with adjustable $l / d$. This is realized using three separate coils with different $l / d$ values close to the theoretical optimum, which can be selectively activated to find the optimal shielding factor. Coils can also be activated in pairs or all together; however, the mutual inductance between active coils means that their combined shielding factor is not the product of their individual shielding factors. Since the manufacturing imperfections discussed above lead to an uncertainty in the mutual inductance, it is, at the current manufacturing tolerances of the system, difficult to predict the performance of the coil combinations, a problem that we may overcome in future iterations. The ability to adjust $l / d$ results in a larger volume with a high shielding factor and gives the possibility of counteracting the effect of a common tilt between the magnet axis and the SSC axis. This improved SSC system also allows considerable flexibility in a multitrap electrode structure [4,27-29], where each trap can have its own optimized SSC, activated when particles are in that particular trap.

\section{COIL DESIGN AND CONSTRUCTION}

The coil system is presented in Fig. 3 and consists of three individual single-layer closed solenoids of lengths 68 $\mathrm{mm}, 75 \mathrm{~mm}$, and $86 \mathrm{~mm}$. These are chosen to span a range of $S^{-1}= \pm 0.03$ around an experimentally constrained optimum. Each solenoid is densely wound with Formvarinsulated single-filament $\mathrm{NbTi}$ wire with an uninsulated diameter of $125 \mu \mathrm{m}$. The coils are wound around a cylindrical cryogenic vacuum chamber with an outer diameter of $77 \mathrm{~mm}$. The vacuum chamber is made out of oxygenfree high-conductivity electrolytic copper and contains the stack of Penning-trap electrodes. A cross-section view of this trap installed in our superconducting magnet is shown in Fig. 3(a) and a closer view of the trap can and trap electrodes is shown in Fig. 3(b). Due to experimental constraints, the center of the coils is displaced by $11 \pm 2 \mathrm{~mm}$ from the central ring electrode of the highly sensitive magnetically homogeneous measurement trap, called the precision trap (PT). The solenoids are separated by layers of tightly wound polytetrafluoroethylene (PTFE) tape and a layer of $100 \mu \mathrm{m}$ Kapton foil to provide electrical insulation between the layers and good thermal contact, as illustrated in Fig. 3(c). Each solenoid has its individual quench heater, formed by wrapping part of the $\mathrm{NbTi}$ wire densely around a $100 \Omega$ resistor. By dissipating $0.8 \mathrm{~mW}$ across the quench heater, the $\mathrm{NbTi}$ wire can be heated above its superconducting transition temperature (approximately $9.5 \mathrm{~K}$ ), making sure no persistent currents will flow through the coil. Depending on the dissipated power, the time constants for the on-off switching are on the order of a few seconds. In order to prevent cross talk between different coils and quench heaters, the heaters are physically separated from each other. The heaters are thermally isolated from the copper chamber using multiple layers of Kapton strips with a total thickness of $1.5 \mathrm{~mm}$. The (a)

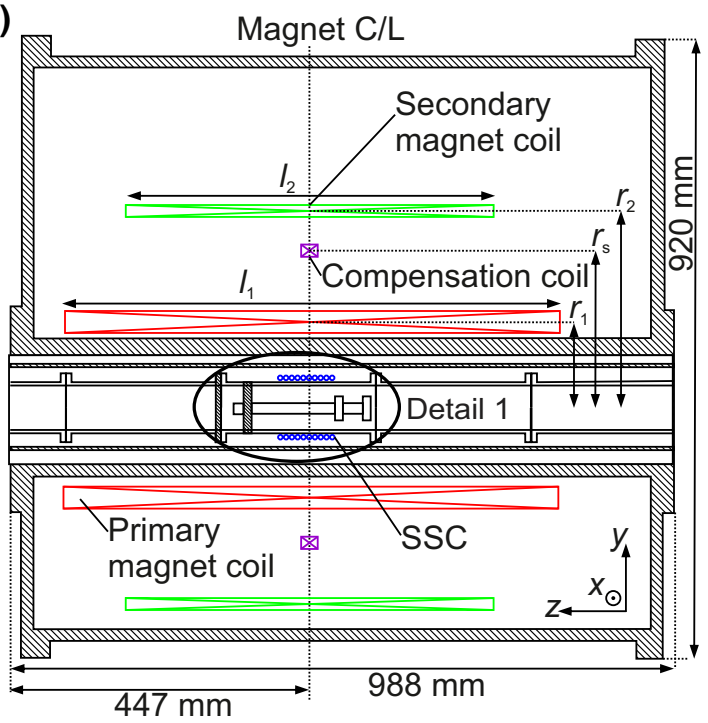

(b) Detail $1 \quad S S C$ C/L

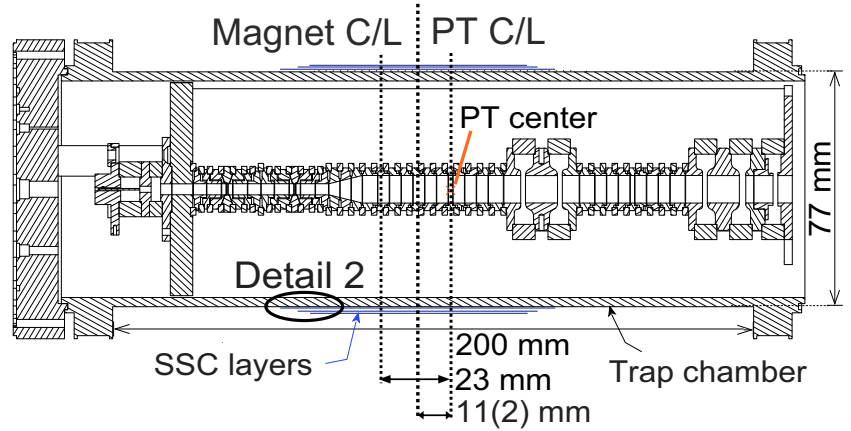

(c) Detail 2

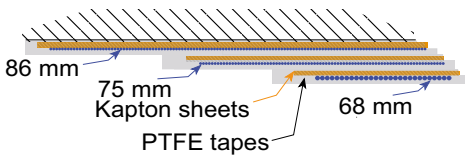

(d)

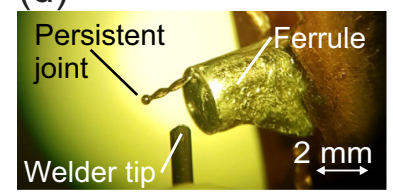

FIG. 3. (a) A schematic of the BASE superconducting magnet with one possible arrangement of its (unknown) coils. (b) A detailed view of the trap chamber and the $68-\mathrm{mm}, 75-\mathrm{mm}$, and $86-\mathrm{mm}$ self-shielding solenoids. Various center lines (C/L) are also shown. (c) An enlarged image showing the isolation of the individual solenoids with layers of Kapton foil and PTFE tape. (d) The persistent joint after welding. 
persistent joints are made with an argon-ion arc-discharge spot welder (Lampert, PUK-5). A photograph of a persistent joint taken through the spot welder's microscope is shown in Fig. 3(d).

\section{MEASURING THE EFFECTIVE SHIELDING FACTOR}

To characterize the effective shielding factor of the SSC system, we apply a set of external magnetic field changes and measure the magnetic field seen by an antiproton kept at the center of the BASE precision trap. By repeating this procedure for each quench configuration of the SSC system, we can determine the intrinsic shielding factor $S$ and misalignment angles $\varepsilon$ and $\theta$ for each of the three coils. The magnetic field at the center of the trap is determined using frequency measurements performed with a single trapped antiproton. In the combined magnetic and electric fields of the Penning trap, the trapped antiproton has three eigenfrequencies, one corresponding to its axial motion with frequency $v_{z} \approx 640.430 \mathrm{kHz}$ and two associated with its radial motion, the modified cyclotron frequency $v_{+} \approx 29.643 \mathrm{MHz}$, and the magnetron frequency $v_{-} \approx 6.917 \mathrm{kHz}$. The invariance theorem [30] relates these frequencies to the free cyclotron frequency $v_{c}$ and hence the total magnetic field $B_{\text {tot }}$ at the center of the Penning trap:

$$
v_{c}=\sqrt{v_{+}^{2}+v_{z}^{2}+v_{-}^{2}}=\frac{1}{2 \pi} \frac{q}{m} B_{\mathrm{tot}} .
$$

The frequencies are measured by detecting induced image currents on the trap electrodes, which are amplified using a tuned $L C$ circuit and cryogenic amplifiers. In the precision trap, two detection circuits are used, one to measure the axial oscillation frequency $v_{z}[12]$ and the other for direct measurements of the modified cyclotron frequency $v_{+}$ [31]. We perform modified cyclotron-frequency measurements using both the sideband frequency-determination method using the axial detector [32] and by directly measuring the modified cyclotron peak frequency at low cyclotron excitation energies $(4-6 \mathrm{eV})$ using the $v_{+}$ detector.

In order to measure the intrinsic shielding factor and the misalignment angles introduced in Eq. (4), we generate defined magnetic field disturbances, record the changes of the external field $\Delta \mathbf{B}_{e}$, and measure the resulting changes in the cyclotron frequency $\Delta v_{c}$ of the trapped antiproton. We produce the magnetic field disturbances by moving two bridge cranes through the experimental hall and simultaneously measuring all three components of the magnetic field in the BASE zone using a sensitive flux-gate magnetometer (Stefan Mayer Instruments FLC3-70). The flux gate is displaced $0.8 \mathrm{~m}$ horizontally along $x$ from the center of the Penning trap, but is otherwise aligned with the trap center. The magnetic field as a function of crane position
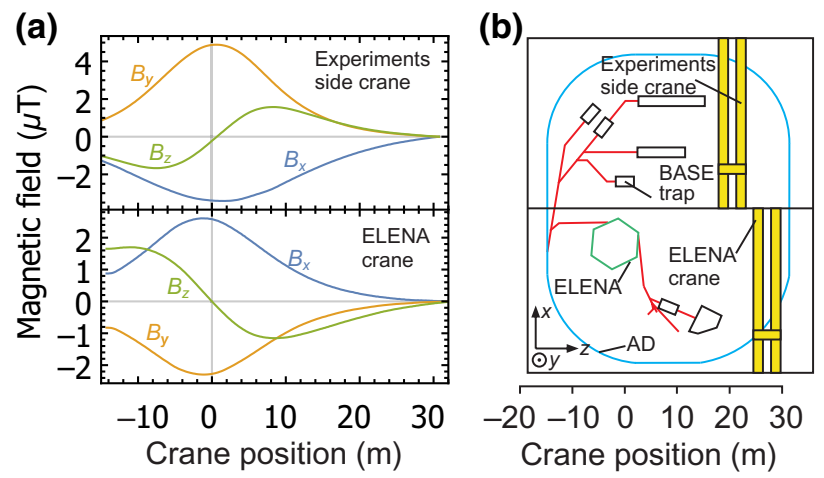

FIG. 4. (a) The measured magnetic field change caused by the two cranes as they are moved over the BASE zone. (b) A plan of the $\mathrm{AD}$ hall, showing the decelerator ring (blue), the antiproton transfer lines (red), the ELENA decelerator (green), and the BASE trap magnet. For the measurements presented here, the experiment-side and ELENA-side bridge cranes (yellow) are only moved along $z$.

in the $\mathrm{AD}$ hall is shown in Fig. 4(a). Figure 4(b) shows the position of the cranes in relation to the BASE experiment and other experimental zones in the AD hall. The intrinsic shielding factor $S$ and misalignment angles $\varepsilon$ and $\theta$ are then extracted by a multivariate fit of the following function:

$$
\Delta v_{c}=\frac{q\left[S^{-1} \Delta B_{e, z}-\varepsilon\left(\Delta B_{e, x} \cos \theta+\Delta B_{e, y} \sin \theta\right)\right]}{2 \pi m},
$$

where $\Delta v_{c}$ is the change in the cyclotron frequency and $\Delta B_{e, x}, \Delta B_{e, y}$, and $\Delta B_{e, z}$ are provided by the averaged fluxgate readings. Here, we keep only the dominant terms from Eq. (4). In these data, we do not remove the effect of the extra shielding $S=1.2(1)$ provided by the main magnet.

To illustrate the sensitivity of our measurement procedure to each parameter, we show in Fig. 5 the response of the antiproton cyclotron frequency to various types of external magnetic field changes. In this case, only the 86$\mathrm{mm}$ coil is superconducting. Within the error bars, the fitted model, plotted in red, reproduces the changes in the magnetic field measured with the antiproton.

By performing such measurements for each coil, we obtain the respective intrinsic shielding factors and misalignment angles. The shielding characteristics of combinations of coils are investigated as well, but only the combination with the best shielding factor is fully characterized. The results are summarized in Table I.

We first consider the intrinsic shielding factors $S$ for the individual coils. Figure 6 shows the intrinsic shielding factors of the single coils compared to our theoretical calculations as a function of $l / d$. The measured shielding factors show the same trend as the theoretical predictions. Comparing to the calculations, the measured intrinsic shielding factors indicate that the coils are about $2 \mathrm{~mm}$ closer to the 

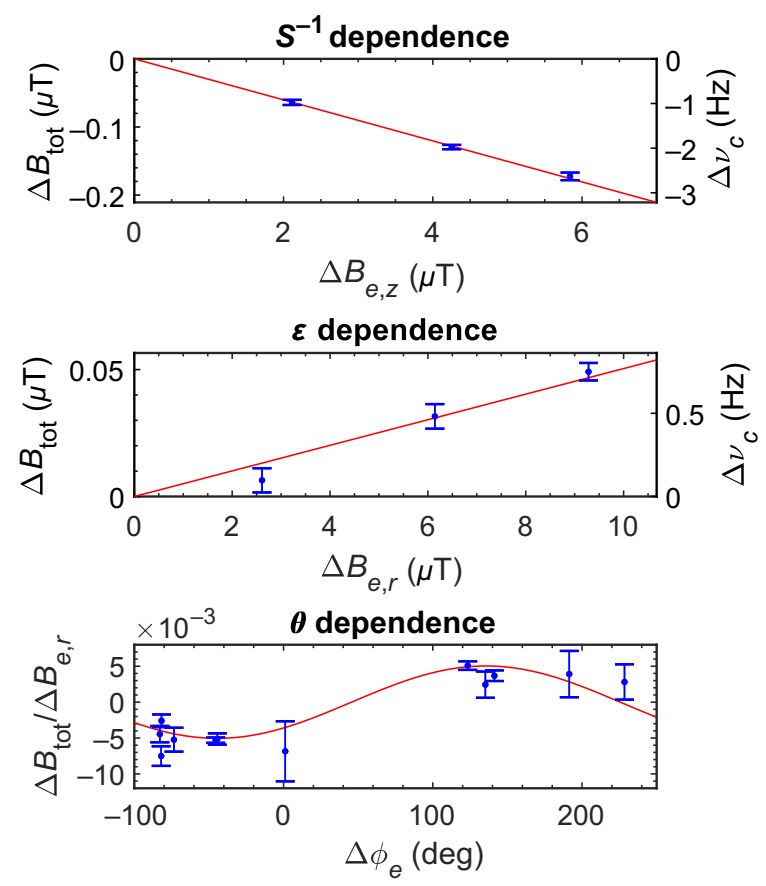

FIG. 5. The magnetic field change registered by the antiproton when the 86-mm SSC is active is shown as a function of several external magnetic field disturbances. The intrinsic shielding factor $S$ governs the response to a field purely along the magnet axis $\Delta B_{e, z}$ (top). The dependence on $\varepsilon$ is visible when the external-field change is purely radial and at a fixed angle $\Delta \phi_{e}=\tan ^{-1}\left(\Delta B_{e, y} / \Delta B_{e, x}\right)$ (middle). When the field is purely radial but its direction $\Delta \phi_{e}$ is varied, the effective shielding factor $S_{\text {eff }}$ is sensitive to the misalignment angle $\theta$ (bottom). The red lines show the behavior predicted by Eq. (1) using the fitted parameters.

PT center than naively expected. This discrepancy could also be attributed to the interaction of the SSC system with the shim coils of the superconducting magnet. Nevertheless, our adjustable self-shielding three-solenoid system achieves a shielding factor of $-210>S>-240$ for fields along the magnet axis by activating the $75-\mathrm{mm}$ coil. While this is one of the largest reported increases in shielding factor achieved by incorporating an SSC system into an existing Penning-trap solenoid, field changes not along the SSC axis will be suppressed to a lesser extent due to the misalignment of the SSC system.

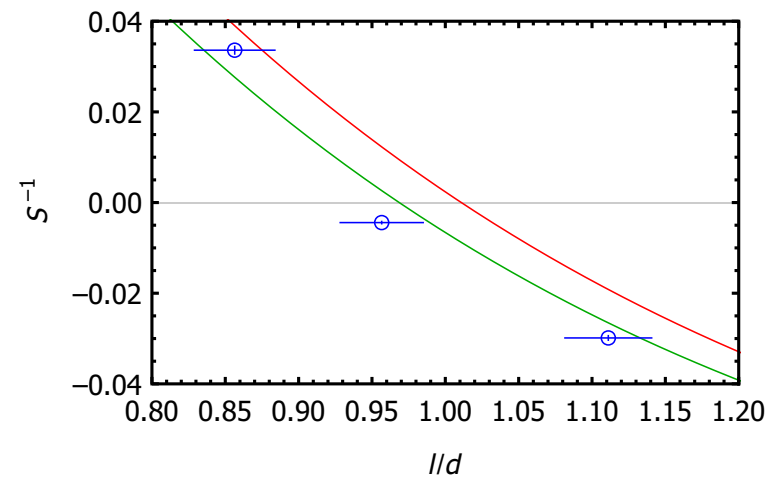

FIG. 6. The inverse intrinsic shielding factors $S^{-1}$ of the individual coils compared to the theoretical predictions. The three coils are plotted according to their value of $l / d$, with the error bars coming from uncertainties in the coil dimensions. The red line plots $S^{-1}$ at the calculated particle position treating the coils as ideal densely wound single-layer solenoids and includes the effects of the mutual inductance with the main solenoid magnet, whereas the green line shows both the effect of the external magnet and a 2-mm displacement of the coil along $z$.

As shown in Table I, the misalignment angles $\varepsilon$ between the coil axis and the BASE magnet are in the range of $0.2^{\circ}-0.5^{\circ}$ for the three coils: these values are consistent with mechanical misalignment and manufacturing accuracy. The last two columns of Table I show the estimated and measured shielding factors for the disturbances imposed by the AD machine. The results are obtained by recording direct cyclotron peak signals [31] at a cyclotronfrequency sampling rate of $0.0625 \mathrm{~Hz}$. Since the magnetic field disturbance is not perfectly aligned with the magnet axis, the shielding performance of the coils for this particular field direction is modified according to Eq. (4). As shown in the table, the measured effective shielding factors are in agreement with what we predict using the measured coil parameters and the known AD field direction, confirming the consistency of our shielding factor model.

\section{PERFORMANCE DURING FREQUENCY MEASUREMENTS}

Figure 7 illustrates different aspects of the performance of the multicoil magnetic shielding system with the $75-\mathrm{mm}$ and $86-\mathrm{mm}$ coils active. In Fig. 7(a), we show a 24-h

TABLE I. The inverse shielding factors and misalignment angles for the three coils and the combination of the 75-mm and 86-mm coils. Based on these parameters, the estimated effective shielding factor for the magnetic field disturbance of the AD cycle is given, together with the measured effective shielding factor during AD operation.

\begin{tabular}{lrlccr}
\hline \hline Coil(s) active $(\mathrm{mm})$ & \multicolumn{1}{c}{$S^{-1}$} & $\varepsilon$ (degrees) & $\theta$ (degrees) & $S_{\text {eff }}^{-1} \mathrm{AD}$, estimated & $S_{\text {eff }}^{-1} \mathrm{AD}$, measured \\
\hline 68 & $0.0336 \pm 8$ & $0.5 \pm 3$ & $183 \pm 14$ & $0.03 \pm 4$ & $0.039 \pm 3$ \\
75 & $-0.0045 \pm 3$ & $0.223 \pm 18$ & $294 \pm 13$ & $-0.059 \pm 6$ & $-0.042 \pm 3$ \\
86 & $-0.0301 \pm 7$ & $0.289 \pm 19$ & $314 \pm 12$ & $-0.07 \pm 3$ & $-0.065 \pm 4$ \\
75 and 86 & $-0.0058 \pm 3$ & $0.267 \pm 21$ & $299 \pm 15$ & $-0.07 \pm 1$ & $-0.071 \pm 5$ \\
\hline \hline
\end{tabular}


(a)
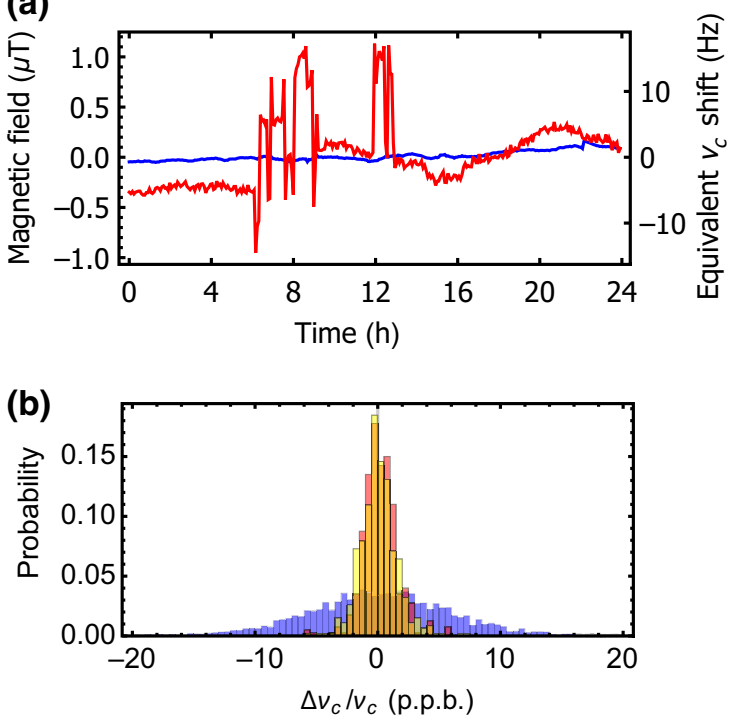

2018-2019: $\square$ AD on: $1.4 \pm 1$ p.p.b. 2014: $5.9 \pm 1$ p.p.b. $A D$ off: $1.5 \pm 1$ p.p.b.

FIG. 7. The performance of the 75-mm and 86-mm coil combination. (a) A comparison of the external magnetic field measured along the magnet axis (red) and the magnetic field measured by the antiproton (blue). (b) The distributions of the pairwise difference of the normalized cyclotron frequency, showing the scatter of the 2014 charge-to-mass ratio measurement with an $S=10$ magnet (blue), the scatter of the current measurement campaign with the SSC system active during AD operation (red), and when the $\mathrm{AD}$ is switched off (yellow).

measurement of the external magnetic field (red) and the magnetic field measured by the antiproton (blue). Due to the high shielding factor of this configuration, the abrupt changes due to craning activities in the facility are strongly suppressed and the experiment continuously samples without any loss of data. To further improve the frequency scatter, we synchronize the cyclotron-frequency measurement cycle to the injection of antiprotons into the decelerator. This ensures that the frequency measurements average over similar AD-imposed magnetic field ramps with a timing jitter of $100 \mu \mathrm{s}$. To quantify the contribution of the magnetic field stability to the ultimate statistical uncertainty that can be reached in our cyclotron-frequency measurements, we record synchronized sequences of cyclotron frequencies $v_{c, k}$, where the subscript $k$ denotes the $k$ th $v_{c}$ measurement, and evaluate the standard deviation $\sigma$ of the recorded fractional frequency differences $\Xi=\sigma\left[\left(v_{c, k+1}-v_{c, k}\right) /\left\langle v_{c}\right\rangle\right]$ as a characteristic figure of merit. Figure 7(b) displays a set of scatter distributions resulting from different measurement campaigns. The blue histogram, with a fractional fluctuation $\Xi_{14} \approx 5.9 \pm 1$ p.p.b., shows results obtained with an $S=10$ magnet in the 2014 antiproton run [1] - the most stringent comparison of antiproton-to-proton charge-to-mass ratios to date. Also shown are two data sets with the self-shielding multicoil system active; the red data are acquired when the $\mathrm{AD}$ is operational and the yellow data during accelerator shutdown. It is clear that there is no significant difference between $\Xi_{18-19, \text { on }} \approx 1.4 \pm 1$ p.p.b. and $\Xi_{18-19, \text { off }} \approx$ $1.5 \pm 1$ p.p.b. Therefore, the magnetic field noise of the $\mathrm{AD}$ is not resolvable in the current experiment due to the performance of the adjustable SSC system. Moreover, the introduction of the multicoil system reduces the cyclotron-frequency fluctuation by a factor of approximately 4 . With the new system, the time needed to reach a given statistical precision during a future antiproton-toproton charge-to-mass ratio measurement is reduced by a factor of 16.

\section{CONCLUSION AND OUTLOOK}

We develop and characterize a multicoil magnetic shielding system that considerably improves the performance of the BASE antiproton experiment in the magnetically noisy environment of the Antiproton Decelerator facility at CERN. The system suppresses external magnetic field fluctuations along the magnet axis by a factor $S=225 \pm 15$, while changes in most other field directions are suppressed by a smaller effective shielding factor $S_{\text {eff. }}$ The system could be further improved by reducing the misalignment angles and by combining the passive SSC with active magnetic field cancellation using external coils, where shielding factors in excess of $10^{4}$ have been observed [33]. These improvements are currently not necessary as with the coil system active and using an accelerator-synchronized frequency sampling method, we reach a fractional cyclotron-frequency scatter of $1.5 \pm 1$ p.p.b., where the suppressed external magnetic field fluctuations are presently not resolved.

The cyclotron-frequency stability is improved by a factor of 4 compared to our previous antiproton-to-proton charge-to-mass ratio comparison [1], which consequently reduces the measurement time needed to reach a certain precision goal by a factor of 16 .

The multicoil SSC system will reduce the line width in antiproton $g$-factor measurements to around 1 p.p.b., which enables sub-parts-per-billion magnetic moment measurements [4], and a further improvement in relative precision by a factor of 10 compared to the result from our recent multitrap measurement $[2,34]$.

Consequently, the multicoil SSC system is an essential tool to improve tests of fundamental symmetries with protons and antiprotons and other Penning-trap precision measurements and may have broader applications to magnetically sensitive measurements in high-field environments.

\section{ACKNOWLEDGMENTS}

We acknowledge technical support from the Antiproton Decelerator group, CERN's cryolaboratory team, and all other CERN groups that provide support to Antiproton 
Decelerator experiments. We acknowledge financial support from the RIKEN Chief Scientist Program, RIKEN Pioneering Project Funding, the RIKEN JRA Program, the Max-Planck Society, the Helmholtz-Gemeinschaft, the DFG through Grant No. SFB 1227 "DQ-mat," the European Union through the Marie Skłodowska-Curie Grant Agreement No. 721559, and the Max-Planck-RIKEN-PTB Center for Time, Constants and Fundamental Symmetries.

J.A.D. and E.W. characterized the coil system, performed all related measurements, and analyzed the data. Both contributed equally to this work. J.A.D., E.W., T.H., J.J.H., and S.U. set up the coil system. S.U. and C.S. designed and built the experiment, J.A.H., M.J.B., T.H., J.A.D., P.E.B., E.W., and S.U. implemented recent technical upgrades. J.A.D., E.W., J.A.H., T.H., M.J.B., P.E.B., S.E., and S.U. contributed to experiment maintenance and operation. All authors read, edited, and approved the paper.

[1] S. Ulmer, C. Smorra, A. Mooser, K. Franke, H. Nagahama, G. Schneider, T. Higuchi, S. Van Gorp, K. Blaum, Y. Matsuda, and W. Quint, High-precision comparison of the antiproton-to-proton charge-to-mass ratio, Nature 524, 196 (2015).

[2] C. Smorra, S. Sellner, M. J. Borchert, J. A. Harrington, T. Higuchi, H. Nagahama, T. Tanaka, A. Mooser, G. Schneider, M. Bohman, K. Blaum, Y. Matsuda, C. Ospelkaus, W. Quint, J. Walz, Y. Yamazaki, and S. Ulmer, A parts-perbillion measurement of the antiproton magnetic moment, Nature 550, 371 (2017).

[3] A. Mooser, S. Ulmer, K. Blaum, K. Franke, H. Kracke, C. Leiteritz, W. Quint, C. C. Rodegheri, C. Smorra, and J. Walz, Direct high-precision measurement of the magnetic moment of the proton, Nature 509, 596 (2014).

[4] G. Schneider, A. Mooser, M. Bohmanm, N. Schön, J. Harrington, T. Higuchi, H. Nagahama, S. Sellner, C. Smorra, K. Blaum, Y. Matsuda, W. Quintm, J. Walzm, and S. Ulmer, Double-trap measurement of the proton magnetic moment at 0.3 parts per billion precision, Science $\mathbf{3 5 8}, 1081$ (2017).

[5] V. M. Shabaev, A. I. Bondarev, D. A. Glazov, M. Y. Kaygorodov, Y. S. Kozhedub, I. A. Maltsev, A. V. Malyshev, R. V. Popov, I. I. Tupitsyn, and N. A. Zubova, Stringent tests of QED using highly charged ions, Hyperfine Interact. 239, 60 (2018).

[6] F. Heiße, F. Köhler-Langes, S. Rau, J. Hou, S. Junck, A. Kracke, A. Mooser, W. Quint, S. Ulmer, G. Werth, K. Blaum, and S. Sturm, High-Precision Measurement of the Proton's Atomic Mass, Phys. Rev. Lett. 119, 033001 (2017).

[7] E. Myers, High-precision atomic mass measurements for fundamental constants, Atoms 7, 37 (2019).

[8] S. Sturm, F. Köhler, J. Zatorski, A. Wagner, Z. Harman, G. Werth, W. Quint, C. H. Keitel, and K. Blaum, Highprecision measurement of the atomic mass of the electron, Nature 506, 467 (2014).
[9] D. Hanneke, S. Fogwell, and G. Gabrielse, New Measurement of the Electron Magnetic Moment and the Fine Structure Constant, Phys. Rev. Lett. 100, 120801 (2008).

[10] H. G. Dehmelt, Radiofrequency spectroscopy of stored ions I: Storage, Adv. At. Mol. Phys. 3, 53 (1968).

[11] S. Sellner, M. Besirli, M. Bohman, M. J. Borchert, J. Harrington, T. Higuchi, A. Mooser, H. Nagahama, G. Schneider, C. Smorra, T. Tanaka, K. Blaum, Y. Matsuda, C. Ospelkaus, W. Quint, J. Walz, Y. Yamazaki, and S. Ulmer, Improved limit on the directly measured antiproton lifetime, New J. Phys. 19, 083023 (2017).

[12] H. Nagahama, G. Schneider, A. Mooser, C. Smorra, S. Sellner, J. Harrington, T. Higuchi, M. Borchert, T. Tanaka, M. Besirli, K. Blaum, Y. Matsuda, C. Ospelkaus, W. Quint, J. Walz, Y. Yamazaki, and S. Ulmer, Highly sensitive superconducting circuits at $\sim 700 \mathrm{kHz}$ with tunable quality factors for image-current detection of single trapped antiprotons, Rev. Sci. Instrum. 87, 113305 (2016).

[13] S. Rainville, J. K. Thompson, E. G. Myers, J. M. Brown, M. S. Dewey, E. G. Kessler, R. D. Deslattes, H. G. Börner, M. Jentschel, P. Mutti, and D. E. Pritchard, World year of physics: A direct test of $E=m c 2$, Nature 438, 1096 (2005).

[14] E. G. Myers, The most precise atomic mass measurements in Penning traps, Int. J. Mass Spectrom. 349-350, 107 (2013).

[15] S. Maury, The antiproton decelerator: AD, Hyperfine Interact. 109, 43 (1997).

[16] M. J. G. Borge and K. Blaum, Focus on exotic beams at ISOLDE: A laboratory portrait, J. Phys. G Nucl. Part. Phys. 45, 010301 (2018).

[17] M. Block, D. Ackermann, D. Beck, K. Blaum, M. Breitenfeldt, A. Chauduri, A. Doemer, S. Eliseev, D. Habs, S. Heinz et al., The ion-trap facility SHIPTRAP, Eur. Phys. J. A 25, 49 (2005).

[18] T. Eronen, V. S. Kolhinen, V.-V. Elomaa, D. Gorelov, U. Hager, J. Hakala, A. Jokinen, A. Kankainen, P. Karvonen, S. Kopecky, I. D. Moore, H. Penttilä, S. Rahaman, S. RintaAntila, J. Rissanen, A. Saastamoinen, J. Szerypo, C. Weber, and J. Äystö, JYFLTRAP: A Penning trap for precision mass spectroscopy and isobaric purification, Eur. Phys. J. A 48, 46 (2012).

[19] J. Dilling, R. Baartman, P. Bricault, M. Brodeur, L. Blomeley, F. Buchinger, J. Crawford, J. R. Crespo López-Urrutia, P. Delheij, M. Froese et al., Mass measurements on highly charged radioactive ions, a new approach to high precision with TITAN, Int. J. Mass Spectrom. 251, 198 (2006).

[20] R. Ringle, P. Schury, T. Sun, G. Bollen, D. Davies, J. Huikari, E. Kwan, D. J. Morrissey, A. Prinke, J. Savory, S. Schwarz, and C. Sumithrarachchi, Precision mass measurements with LEBIT at MSU, Int. J. Mass Spectrom. 251, 300 (2006).

[21] R. C. Pardo, G. Savard, and R. V. F. Janssens, ATLAS with CARIBU: A laboratory portrait, Nucl. Phys. News 26, 5 (2016).

[22] H.-J. Kluge, T. Beier, K. Blaum, L. Dahl, S. Eliseev, F. Herfurth, B. Hofmann, O. Kester, S. Koszudowski, C. Kozhuharov et al., HITRAP: A facility at GSI for highly charged ions, Adv. Quantum Chem. 53, 83 (2008). 
[23] C. Smorra, K. Blaum, L. Bojtar, M. Borchert, K. A. Franke, T. Higuchi, N. Leefer, H. Nagahama, Y. Matsuda, A. Mooser, M. Niemann, C. Ospelkaus, W. Quint, G. Schneider, S. Sellner, T. Tanaka, S. Van Gorp, J. Walz, Y. Yamazaki, and S. Ulmer, BASE - The baryon antibaryon symmetry experiment, Eur. Phys. J. Spec. Top. 224, 3055 (2015).

[24] W. Bartmann, P. Belochitskii, H. Breuker, F. Butin, C. Carli, T. Eriksson, W. Oelert, R. Ostojic, S. Pasinelli, and G. Tranquille, The ELENA facility, Phil. Trans. R. Soc. A 376, 20170266 (2018).

[25] G. Gabrielse and J. Tan, Self-shielding superconducting solenoid systems, J. Appl. Phys. 63, 5143 (1988).

[26] L. Cohen, An Exact Formula for the Mutual Inductance of Coaxial Solenoids, Bulletin of the Bureau of Standards (Govt. Print. Off., Washington, 1907).

[27] H. Häffner, T. Beier, S. Djekić, N. Hermanspahn, H.-J. Kluge, W. Quint, S. Stahl, J. Verdú, T. Valenzuela, and G. Werth, Double Penning trap technique for precise $g$ factor determinations in highly charged ions, Eur. Phys. J. D 22, 163 (2003).

[28] A. Mooser, S. Bräuninger, K. Franke, H. Kracke, C. Leiteritz, C. C. Rodegheri, H. Nagahama, G. Schneider, C. Smorra, K. Blaum, Y. Matsuda, W. Quint, J. Walz, Y. Yamazaki, and S. Ulmer, Demonstration of the double Penning trap technique with a single proton, Phys. Lett. B 723, 78 (2013).
[29] J. Repp, C. Böhm, J. C. López-Urrutia, A. Dörr, S. Eliseev, S. George, M. Goncharov, Y. N. Novikov, C. Roux, S. Sturm, S. Ulmer, and K. Blaum, PENTATRAP: A novel cryogenic multi-Penning-trap experiment for highprecision mass measurements on highly charged ions, Appl. Phys. B 107, 983 (2012).

[30] L. S. Brown and G. Gabrielse, Precision spectroscopy of a charged particle in an imperfect Penning trap, Phys. Rev. A 25, 2423 (1982).

[31] S. Ulmer, K. Blaum, H. Kracke, A. Mooser, W. Quint, C. C. Rodegheri, and J. Walz, A cryogenic detection system at 28.9 MHZ for the non-destructive observation of a single proton at low particle energy, Nucl. Instrum. Meth. A 705, 55 (2013).

[32] E. A. Cornell, R. M. Weisskoff, K. R. Boyce, and D. E. Pritchard, Mode coupling in a Penning trap: $\pi$ pulses and a classical avoided crossing, Phys. Rev. A 41, 312 (1990).

[33] R. S. Van Dyck, D. L. Farnham, S. L. Zafonte, and P. B. Schwinberg, Ultrastable superconducting magnet system for a Penning trap mass spectrometer, Rev. Sci. Instrum. 70, 1665 (1999).

[34] H. Nagahama, C. Smorra, S. Sellner, M. Besirli, M. Borchert, A. Mooser, G. Schneider, T. Higuchi, T. Tanaka, K. Blaum, Y. Matsuda, C. Ospelkaus, W. Quint, J. Walz, Y. Yamazaki, and S. Ulmer, Sixfold improved single particle measurement of the magnetic moment of the antiproton, Nature Commun. 8, 14084 (2017). 